Revista cientifica de investigación actualización del mundo de las ciencias

Carlos Fernando Morales Vera a ${ }^{\text {a }}$ Lourdes Ximena Reyes Suárez ${ }^{\text {b; }}$ Mariana Noemi Medina Suarez ${ }^{c}$; Amir Ricardo Villon Cruz ${ }^{\mathrm{d}}$

Competencias digitales en docentes: desafío de la educación superior

Digital competences in teachers: challenge of higher education

Revista Científica de Investigación actualización del mundo de las Ciencias. Vol. 3

núm., 3, julio, ISSN: 2588-0748, 2018, pp. 1006-1034

DOI: 10.26820/reciamuc/3.(3).julio.2019.1006-1034

URL: http://reciamuc.com/index.php/RECIAMUC/article/view/313

Código UNESCO: 33 Ciencias Tecnológicas

Tipo de Investigación: Artículo de Revisión

(c) RECIAMUC; Editorial Saberes del Conocimiento, 2019

Recibido: 28/04/2019

Aceptado: 19/05/2019

Publicado: 01/07/2019

Correspondencia: fernando.morales@educacion.gob.ec

a. Magister en Diseño y Evaluación de Modelos Educativos; Licenciado en Ciencias de la Educación Mención Administración Educativa; Ministerio de Educación; Guayaquil, Ecuador; fernando.morales@educacion.gob.ec

b. Ingeniera en Administración de Empresas; Ministerio de Educación; Guayaquil, Ecuador; ximena.reyes@educación.gob.ec

c. Magister en Diseño y Evaluación de Modelos Educativos; Licenciada en Ciencias de la Educación Especialización Educación Primaria; Profesora Especialización Educación Primaria; Ministerio de Educación; Guayaquil, Ecuador; marianoem1@ hotmail.com

d. Licenciado en Administración Publica; Investigador Independiente; Guayaquil, Ecuador; villoncruzamir@gmail.com 


\section{Competencias digitales en docentes: desafío de la educación superior}

Vol. 3, núm. 3., (2019)

Carlos Fernando Morales Vera; Lourdes Ximena Reyes Suárez; Mariana Noemi Medina Suarez;

Amir Ricardo Villon Cruz

\section{RESUMEN}

La evolución de la sociedad ha tenido como característica el crecimiento del conocimiento. Esta adquisición del saber ha permitido que la humanidad garantice sus necesidades básicas. Uno de los espacios donde se sigue fomentando el desarrollo del conocimiento son las universidades. Dentro de estas, la forma como se imparte el conocimiento ha estado arraigada a una cultura totalmente conductista donde una persona es la dueña del conocimiento y su función es impartirlo a un grupo de personas, en este caso estudiantes. En este mundo globalizado, el conocimiento esta desarrollado por todo aquel que hace vida dentro del aula de clase y esto ocurre por el avance de las tecnologías de la información y comunicación. El miedo al cambio, el conformismo, salir de la zona de confort, entre otros, son los parámetros subjetivos dentro de la docencia, las cuales no permiten que las nuevas herramientas pedagógicas pueden ayudar a fomentar una nueva cultura dentro del claustro universitario. Por lo cual, el objetivo general de esta investigación es analizar las competencias digitales en docentes como desafío de la educación superior. La metodología empleada se basó en investigaciones de tipo documental y bibliográfica. Los resultados obtenidos se basaron en las TIC como herramientas en la educación superior en Ecuador, las herramientas digitales innovadoras en la educación superior y la relación de las TIC y los docentes en la educación superior. Como conclusión se obtuvo que ha comenzado, de manera lenta, la transformación de la pedagogía a nivel superior, la cual permitirá la generación de conocimiento de manera rápida y eficiente con la ayuda de las herramientas digitales permitiendo que las competencias en los docentes sea factible, ya que el profesor tendrá en su poder apegarse al cambio que se está viviendo en este mundo globalizado, así como también cuente con el apoyo en equipo e infraestructura por parte de los institutos de educación superior.

Palabras Claves: Conocimiento; Pedagogía; TIC; Herramientas Digitales. 


\title{
Competencias digitales en docentes: desafío de la educación superior
}

Vol. 3, núm. 3., (2019)

Carlos Fernando Morales Vera; Lourdes Ximena Reyes Suárez; Mariana Noemi Medina Suarez; Amir Ricardo Villon Cruz

\begin{abstract}
The evolution of society has had as a characteristic the growth of knowledge. This acquisition of knowledge has allowed humanity to guarantee its basic needs. One of the spaces where the development of knowledge continues to be promoted are universities. Within these, the way in which knowledge is imparted has been rooted in a totally conductivist culture where a person is the owner of knowledge and its function is to impart it to a group of people, in this case students. In this globalized world, knowledge is developed by everyone who lives within the classroom and this occurs through the advancement of information and communication technologies. The fear of change, conformism, leaving the comfort zone, among others, are the subjective parameters within the teaching, which do not allow the new pedagogical tools can help to foster a new culture within the university cloister. Therefore, the general objective of this research is to analyze the digital competences in teachers as a challenge of higher education. The methodology used was based on documentary and bibliographic research. The results obtained were based on ICT as tools in higher education in Ecuador, innovative digital tools in higher education and the relation of ICT and teachers in higher education. As a conclusion, it was obtained that the transformation of pedagogy at a higher level has begun slowly, which will allow the generation of knowledge quickly and efficiently with the help of digital tools, allowing competences in teachers to be feasible. that the professor will be in his power to be attached to the change that is being lived in this globalized world, as well as to count on the support in equipment and infrastructure on the part of the institutes of superior education.
\end{abstract}

Key Words: Knowledge; Pedagogy; ICT; Digital Tools. 


\section{Competencias digitales en docentes: desafío de la educación superior}

Vol. 3, núm. 3., (2019)

Carlos Fernando Morales Vera; Lourdes Ximena Reyes Suárez; Mariana Noemi Medina Suarez;

Amir Ricardo Villon Cruz

\section{Introducción.}

Una de las características del proceso evolutiva de la humanidad fue la necesidad de poder garantizar las necesidades básicas por lo que el hombre tuvo que buscar e indagar con técnicas que fueron descubiertas de improvisto o por curiosidad de saber que pasaba si alteraba o modificaba una situación. Por supuesto, nace la investigación como un fenómeno intrínseco en el ser humano. Investigar es un proceso inherente a la vida misma; no obstante, la investigación en educación con lleva, además de un interés y una necesidad, una búsqueda para la profundización y comprensión de los fenómenos educativos, más allá de lo meramente evidente. Involucra una búsqueda(Pereira Pérez, 2011).

La investigación, de manera formal, se analiza y comprende en los diferentes niveles de educación por la cual se enfrenta un individuo en su desarrollo académico. En estas etapas se formaliza las técnicas y métodos que se utilizan para poder encontrar la solución a diferentes problemáticas aplicando una investigación desde diferentes aristas. La investigación se puede definir como el efecto de realizar actividades intelectuales y experimentales de modo sistemático con el propósito de aumentar los conocimientos sobre una determinada materia y teniendo como fin ampliar el conocimiento científico, sin perseguir, en principio, ninguna aplicación práctica(Ortiz Flores \& Bernal Zepeda, 2007).

Ahora, el proceso investigativo tiene un fundamento por la cual se desarrolla, basándose en las necesidades y en los objetivos del trabajo. Este fundamento se desarrolla a través de un procedimiento metodológico. Para realizar un trabajo de investigación es necesario llevar a cabo un desarrollo metódico que permita la adecuada consecución de los objetivos propuestos, así 


\section{Competencias digitales en docentes: desafío de la educación superior}

Vol. 3, núm. 3., (2019)

Carlos Fernando Morales Vera; Lourdes Ximena Reyes Suárez; Mariana Noemi Medina Suarez; Amir Ricardo Villon Cruz

como una formulación concreta del problema y una metodología de investigación rigurosa y adecuada al tipo de trabajo(López Noguero, 2002).

Es por ello, que es necesario poder reconocer los elementos que conforman la investigación para si poder desarrollar metodológicamente la solución a las diversas interrogantes que se plantean al momento de solventar una situación.

"Se reconocen cuatro elementos presentes en toda investigación: sujeto, objeto, medio y fin. De esta manera, se entiende por sujeto el que desarrolla la actividad, el investigador; Por objeto, lo que se investiga, esto es, la materia o el tema; Por medio, lo que se requiere para llevar a cabo la investigación, es decir, el conjunto de métodos y técnicas; Por fin, lo que se pretende sean los resultados, los propósitos de la actividad, que radica en la solución de un problema o situación previamente detectados”.(Ortiz Flores \& Bernal Zepeda, 2007, pág. 5)

Es decir, que la investigación se convierte en un factor aglomerante de la integración y desarrollo de la sociedad. El sistema educativo, y en especial la educación superior, es el factor clave para el desarrollo profesional y personal de los individuos que se convertirán en el sostén económico del país, ya que en ella se fomenta la generación del conocimiento. La universidad debe fomentar el espíritu de investigación en todos los niveles de la formación académica, propiciando la formación integral de sus estudiantes de pregrado y postgrado, con la participación activa del claustro de profesores(Carrizo Estévez, 2010).

Dentro del claustro universitario se encuentran varios parámetros las cuales permitirán que este desarrollo se desenvuelva eficientemente y así determine el desarrollo profesional de la sociedad. Estos parámetros están regidos, en su mayoría por paradigmas incrustados dentro del 


\section{Competencias digitales en docentes: desafío de la educación superior}

Vol. 3, núm. 3., (2019)

Carlos Fernando Morales Vera; Lourdes Ximena Reyes Suárez; Mariana Noemi Medina Suarez;

Amir Ricardo Villon Cruz

ambiente académico. Un paradigma engloba un sistema de creencias sobre la realidad, la visión del mundo, el lugar que el individuo ocupa en él y las diversas relaciones que esa postura permitiría con lo que se considera existente (Ramos, 2015).

Los paradigmas históricos dentro de la educación son variados, van desde el manejo de los recursos económicos hasta el desarrollo de actividades educativas y culturales. Pero más significativamente, los paradigmas están en la metodología pedagógica utilizadas por los docentes de las diferentes asignaturas de las universidades. Se rigen bajo paradigmas positivistas, conductistas o constructivistas, entre otros. Esto requiere que existan un cambio dentro de esta cultura y poder producir una implosión dentro de la educación. Es por ello, que ahí la necesidad de prestar mayor atención en los procesos de formación y capacitación docente a este tema como una de las condiciones fundamentales a la hora de diseñar y elaborar los correspondientes dispositivos pedagógico didácticos (Zaccagnini, 2003).

Aquí recae la responsabilidad desde las autoridades de las instituciones de educación superior hasta los representantes gubernamentales a nivel regional y nacional. Se debe crear nuevos paradigmas basados en sus propias creencias, culturas, necesidades y capacidades. Este paradigma consistiría básicamente en una enseñanza de las ciencias acorde con la propia metodología científica, la cual a menudo ha sido mal entendida por los docentes que han asumido visiones muy simplistas, marcadas por un positivismo extremo(GIL PEREZ, 1983).

Esto permitiría un auge del desarrollo del conocimiento y por ende una mayor calidad en los profesionales egresados de las universidades. Lo que si se debe hacer es cambiar la cultura dentro de las áreas académicas y administrativas que permitan formar docentes con nuevas 


\section{Competencias digitales en docentes: desafío de la educación superior}

Vol. 3, núm. 3., (2019)

Carlos Fernando Morales Vera; Lourdes Ximena Reyes Suárez; Mariana Noemi Medina Suarez; Amir Ricardo Villon Cruz

visiones pedagógicas aplicando las nuevas herramientas que brindan este mundo globalizado. El reduccionismo técnico-instrumental en que se cae la dinámica curricular de las instituciones formadoras docentes como en la variada oferta de capacitación y perfeccionamiento, solo contribuye al sostenimiento de una educación que gravita a partir de la particular concepción del sujeto pedagógico universal (Zaccagnini, 2003).

Esto permite descubrir que el conocimiento no es generado por una sola persona, dentro del aula de clase, sino por los participantes que hacen vida dentro de ella, permitiendo una masificación del mismo. Poder aplicar este nuevo paradigma requiere un cambio también en la cultura universitaria y, por supuesto, del docente. Por lo cual, debe existir un cambio radical en la formación y desarrollo de docentes dentro de las universidades pedagógicas.

“La formación y actualización de los profesionales de la educación superior es indispensable y debe ser tan dinámica como lo son los avances de la ciencia y la tecnología, los cuales no serán aprovechados ni estimados si las instituciones no asumen con urgencia la formación de su recurso humano, a través de programas variados destinados a inducir cambios en sus competencias y en su papel como docentes”.(Briceño, 2014, pág. 72)

Todo esto conlleva a un cambio necesario dentro de la educación superior. La idea esencial del cambio intrínseco se puede sintetizar en la necesidad de cambiar el paradigma educativo desde un modelo basado casi con exclusividad en el conocimiento, a otro sustentado en la formación integral de los individuos(Mora, 2004).Un sistema educativo que promueva la integración efectiva entre las comunidades, el entorno social, con las áreas interdisciplinarias y transdisciplinarias del currículum, por ejemplo, pedagógica, psicológica y social; propiciando 


\section{Competencias digitales en docentes: desafío de la educación superior}

Vol. 3, núm. 3., (2019)

Carlos Fernando Morales Vera; Lourdes Ximena Reyes Suárez; Mariana Noemi Medina Suarez;

Amir Ricardo Villon Cruz

programas de desarrollo integral, con acento en la acción social comunitaria(Acosta \& Genyelbert, 2013).

Es decir, se debe realizar un empoderamiento de la educación en todos los niveles. El conocimiento debe surgir desde las diferentes aristas de la sociedad, pero como factor predominante y como líder de la organización, las universidades. «Empoderar» a quien quiera compartir lo que sabe para encontrarse con aquellos que quieren aprender de él, es por ello que el aprendizaje social y en comunidades logra este objetivo (Reig-Hernández, 2010).

Esto permite un cambio dentro de las complejidades que se viven dentro de las universidades permitiendo sentar nuevas bases. La articulación de los procesos educativos con la innovación, la expansión de saberes, las competencias y las nuevas formas de creación de riqueza con la informatización, rearticulan las relaciones entre educación y trabajo, así como entre educación y desarrollo(Rama, 2010).

Existen herramientas fundamentales para el desarrollo de este empoderamiento y es el creciente desarrollo de las plataformas que conforman la internet. Aquí entra en juego las tecnologías de la información y comunicación (TIC). Son recursos que permiten la interacción directa entre los usuarios facilitando la construcción colectiva de contenidos, intercambiando información de valor o simplemente vinculando intereses o proyectos comunes, que pueden ser potenciados a través de la coordinación de acciones soportadas por plataformas digitales(Martínez Alvarado, 2009).Con estas herramientas se permite un desarrollo eficiente del conocimiento y por ende un crecimiento de la economía del país debido a la formación de profesionales integrales. 


\section{Competencias digitales en docentes: desafío de la educación superior}

Vol. 3, núm. 3., (2019)

Carlos Fernando Morales Vera; Lourdes Ximena Reyes Suárez; Mariana Noemi Medina Suarez; Amir Ricardo Villon Cruz

La falta de integración de las tecnologías digitales en la formación está relacionada con una falta de cambio en las orientaciones pedagógicas necesarias para abordar el conocimiento y las competencias de los alumnos actuales(Gros \& Noguera, 2015). Lamentablemente, existe una falta de interés que repercute en los diferentes niveles organizativos de las instituciones de educación superior para con la interacción de las competencias digitales en la formación del conocimiento y por la cual se transforma en el principal parámetro de desafío para la educación superior. En este marco, el profesorado tiene un importante papel en la orientación y guía para un uso apropiado de la comunicación y las fuentes de información académicas(Escofet Roig, García Gonzalez, \& Gros Salvat, 2011).

Por lo tanto, esta investigación tiene como objetivo general analizar las competencias digitales en docentes como desafío de la educación superior. La metodología empleada se basó en investigaciones de tipo documental y bibliográfica.

\section{Método.}

La metodología empleada se basó en investigaciones de tipo documental y bibliográfica enfocándose en la comprensión de los viejos paradigmas pedagógicos, en el análisis de la importancia de las TIC, en el conocimiento de las herramientas digitales innovadoras y en el diagnóstico de la relación de las TIC y los docentes en la educación superior. Para ello se tomó en consideración herramientas metodológicas, tales como investigaciones documentales y bibliográficas por medio de textos, documentos y artículos científicos publicados disponibles en la web. 


\section{Competencias digitales en docentes: desafío de la educación superior}

Vol. 3, núm. 3., (2019)

Carlos Fernando Morales Vera; Lourdes Ximena Reyes Suárez; Mariana Noemi Medina Suarez;

Amir Ricardo Villon Cruz

\section{Resultados.}

Las TIC como herramientas en la educación superior en Ecuador

Una de las características que deben poseer todas las instituciones de educación superior es un sistema de calidad que permita generar el conocimiento imprescindible para el desarrollo de la sociedad. Según Cabrera (2005) existen características que pueden ayudar a fomentar una mejor calidad universitaria:

- Ser operacional, es decir poder traducirse en elementos fácilmente manejables dentro de unaguía, modelo o procedimiento de evaluación.

- Que abarque de una u otra forma las funciones sustantivas de la universidad.

- Que lleve implícito el concepto de apreciación o evaluación.

- Que esté ligado a la pertinencia social.

Conjuntamente con estas características, el sistema educativo de la primaria y secundaria también debe aportar su cuota para el desarrollo del conocimiento. El Ecuador debe invertir grandes recursos para mejorar la calidad de su educación básica y para implementar programas para mantener a todos los estudiantes en las escuelas y colegios hasta que sean elegibles para asistir a la universidad (Post, 2011). Esto quiere decir que debe haber un cambio de paradigmas y culturas dentro de esos niveles para cuando lleguen los estudiantes estén familiarizados con el cambio.

Con estos cambios permitirá que la educación superior cubra las expectativas de transformación de las sociedades, tomando el protagonismo el estado y los diferentes actores 


\section{Competencias digitales en docentes: desafío de la educación superior}

Vol. 3, núm. 3., (2019)

Carlos Fernando Morales Vera; Lourdes Ximena Reyes Suárez; Mariana Noemi Medina Suarez; Amir Ricardo Villon Cruz

dentro del claustro universitario. Esto último implica que la educación superior debe observar de manera atenta su pertinencia y constituirse en mecanismo privilegiado para el desarrollo nacional y la inserción soberana del Ecuador en el contexto de las naciones(Ramírez \& Minteguiada, 2010).

Poder insertarse dentro de un mundo globalizado conlleva a evaluar las fortalezas y debilidades que pueda tener Ecuador dentro de su sistema de educación superior. Dentro de los beneficios para estos nuevos caminos están la gestión de recursos físicos y humanos que estén dispuesto a efectuar ese paso. Estos son representados por la necesidad de recursos humanos y herramientas de gestión en tecnología e innovación, que permitan dar cuenta de las principales dinámicas del sistema, mejorar la evaluación de los programas e instrumentos promoviendo una adecuación inteligente de los mismos a los objetivos planteados(Milia, 2014).

Ahora las debilidades al cambio fundamentalmente se basan en la lenta adaptación del capital humano para la interacción de los componentes digitales. Estudiantes y Profesores se ven abocados a cambios trascendentales en su forma de enseñar y aprender, sin tener apenas tiempo de reflexión sobre ambos particulares ya que los cambios siempre son graduales, muy a pesar de que las tecnologías cambien de manera desaforada(Quintero, 2009).

Indudablemente que se plantean retos para la generación del conocimiento en este mundo globalizado. Estos desafíos los deben tomar las instituciones de educación superior. Constatando esta realidad, se deben aprovechar laspotencialidades que ofrecen las tecnologías, de modo que se integren estos recursos en losesquemas de trabajo universitario, pero no de cualquier modo(Rodríguez-Izquierdo, 2011).Estos retos en las tecnologías permiten que la educación 


\section{Competencias digitales en docentes: desafío de la educación superior}

Vol. 3, núm. 3., (2019)

Carlos Fernando Morales Vera; Lourdes Ximena Reyes Suárez; Mariana Noemi Medina Suarez; Amir Ricardo Villon Cruz

superior deba plantearse nuevos cambios. Los cambios que hay que realizar son de dos tipos: intrínsecos (del modelo pedagógico) y extrínsecos (del modelo organizativo de las instituciones)(Mora, 2004).

Ya definidas las pautas para realizar estos cambios, entonces es necesario poder encontrar una conceptualización de las TIC y su incrustación en el currículo del docente. Las TIC son el conjunto de tecnologías que permiten el acceso, producción, tratamiento y comunicación de información presentada en diferentes códigos (texto, imagen, sonido)(Ortí, 2011).Con esto se puede diseñar las funciones de las TIC dentro de la educación, como se plantea en la Figura 1. La incorporación de las TIC en la carrera docente se expresa de la siguiente forma:

“Como contenido desde el inicio de la misma, puede ser como: curso propedéutico; asignatura particular, como eje transversal que impregne todo el currículo, con el uso de las TIC (e-mail, Chat, espacios, blog, foros de discusión, uso de ambientes basados en la Web, presentaciones, software educativos entre otros) usando efectivamente, el correo electrónico, el Chat, las presentaciones en PowerPoint y otras herramientas en formato electrónico en los cursos regulares, con el modelaje y la posibilidad de transferencia en otros contextos".(Castro, 2007, pág. 230) 


\section{Competencias digitales en docentes: desafío de la educación superior}

Vol. 3, núm. 3., (2019)

Carlos Fernando Morales Vera; Lourdes Ximena Reyes Suárez; Mariana Noemi Medina Suarez; Amir Ricardo Villon Cruz

- Medio de expresión: escribir, dibujar, presentaciones, webs..

- Canal de comunicación, colaboración e intercambio.

- Instrumento para procesar la información.

- Fuente abierta de información (mass media, self media)

- Instrumento para la gestión administrativa y tutorial.

- Herramienta de diagnóstico y rehabilitación.

- Medio didáctico: informa, entrena, guía aprendizaje, motiva

- Generador de nuevos escenarios formativos

- Medio lúdico y para el desarrollo cognitivo.

- Contenido curricular: conocimientos, competencias

Figura 1. Funciones de las TIC en la educación

Fuente: (Graells, 2013)

Poder incorporar las TIC dentro de la educación superior puede generar que se planteen escenarios que deriven de esta interacción tecnosocial. Dichos posibles escenarios se configuran en torno a dos ejes principales sobre los que pueden tomarse unas u otras decisiones: la centralización o descentralización de los servicios y el peso de la responsabilidad en términos de calidad educativa(Quintero, 2009). Estas se pueden visualizar en la Figura 2. 


\section{Competencias digitales en docentes: desafío de la educación superior}

Vol. 3, núm. 3., (2019)

Carlos Fernando Morales Vera; Lourdes Ximena Reyes Suárez; Mariana Noemi Medina Suarez;

Amir Ricardo Villon Cruz

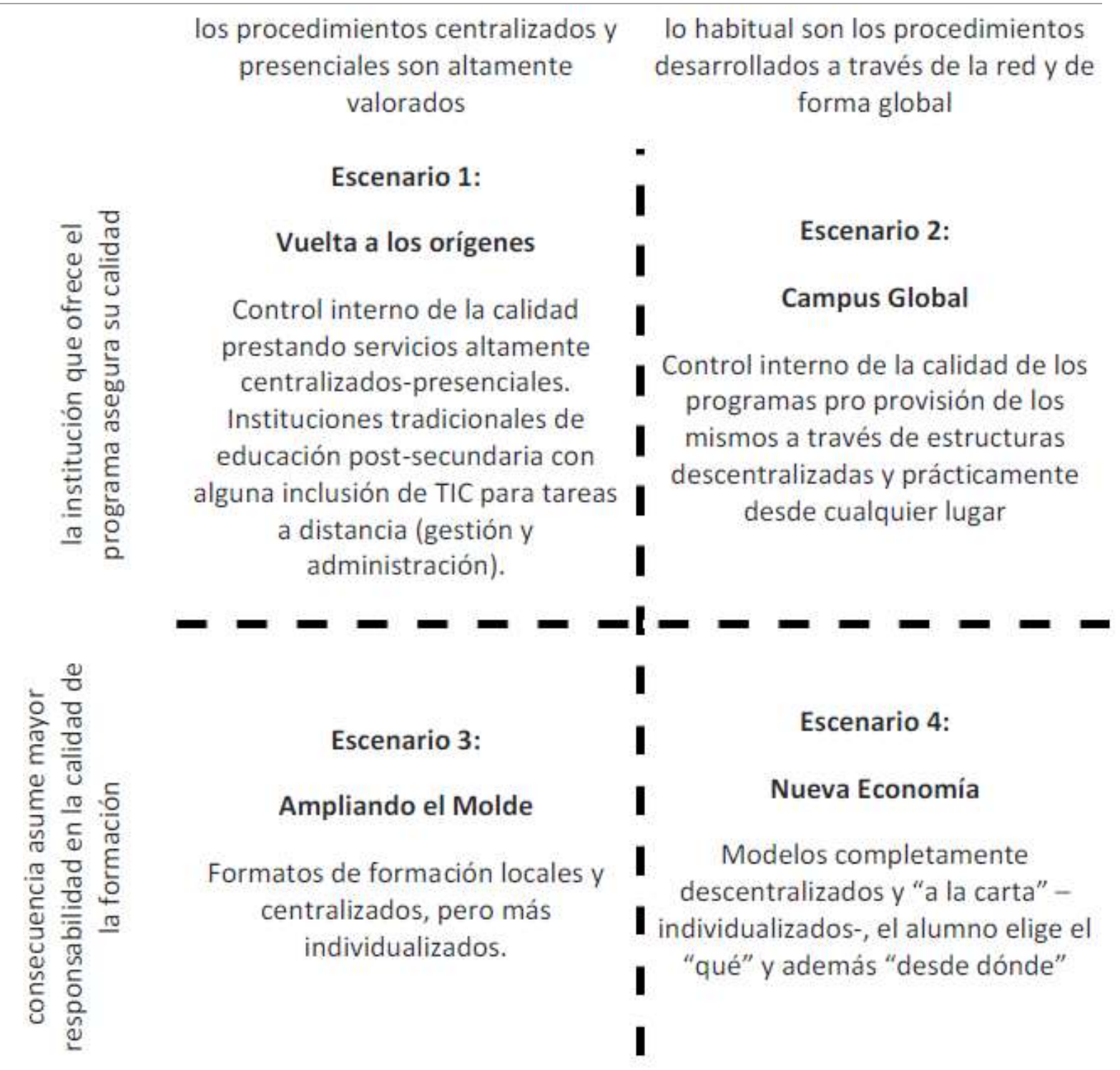

Figura 2. Cuatro escenarios para el desarrollo educativo dentro de las universidades

Fuente: (Quintero, 2009)

Las universidades no plantean adherirse a algunos de estos escenarios sino más buscan una combinación mixta de los mismos. Por lo que, se confirma que la incorporación es compleja, difícil y lenta para moldearse a la cultura universitaria. Los cambios generados por la 


\section{Competencias digitales en docentes: desafío de la educación superior}

Vol. 3, núm. 3., (2019)

Carlos Fernando Morales Vera; Lourdes Ximena Reyes Suárez; Mariana Noemi Medina Suarez; Amir Ricardo Villon Cruz

incorporación de las TIC a la educación no son inmediatos ni fáciles de identificar, por lo que se trata de un proceso complejo que solo da frutos a medio y a largo plazo(Sunkel, 2009).

Lo que si es que se genera una alfabetización digital desde todos los vértices de la sociedad, no solo desde la universidad. No sólo es el aprendizaje del uso funcional de estas tecnologías, sino también el conocimiento de las prácticas socioculturales asociadas al manejo de estas tecnologías en la Sociedad de la Información y la capacidad para participar en esas prácticas utilizándolas de manera adecuada(Coll, 2008).

Todos estos mecanismos generan una virtualidad por lo que se deben aplicar nuevas herramientas pedagógicas y nuevas actitudes hacia estos nuevos aprendizajes. Esta universidad virtual debe entenderse como una infraestructura quebrinde a los estudiantes una experiencia de aprendizajey servicios de apoyo para completar una carrera, y a losmiembros del cuerpo docente, los recursos para enseñare investigar eficazmente en línea(Facundo, 2004).

Herramientas digitales innovadoras en la educación superior

Indudablemente el docente ha buscado y utilizado muchas herramientas pedagógicasque permiten el traslado de la información y por ende la generación del conocimiento. En este mundo globalizado existen muchos vehículos que permiten el traslado en cualquier espacio y tiempo. Todos los esfuerzos, las experiencias y los proyectos colaborativos basados en la incorporación y uso de: plataformas educativas, herramientas de la Web 2.0 y redes sociales deben ser en forma creativa y responsable, propiciando el trabajo en redes colaborativas institucionales(Tamayo, 2012). 


\section{Competencias digitales en docentes: desafío de la educación superior}

Vol. 3, núm. 3., (2019)

Carlos Fernando Morales Vera; Lourdes Ximena Reyes Suárez; Mariana Noemi Medina Suarez; Amir Ricardo Villon Cruz

"Existen innovaciones interesantes que se destacan de acuerdo con los ámbitos: Tecnológico (OCW, MOOC, PCE-GA, mPLE, Gamificación, SeriousGame, WhatsApp, Video). Pedagógico con modelos como (ADIEE y AZZURE) así como metodologías (PACIE y OOHDM) y la Web Social. En cuanto al ámbito institucional se identifican innovaciones como la Integración de sistemas Weblearn de Oxford y Arquitectura de un ecosistema de elearning MIT”.(Guanoluisa \& Herrera, 2018, págs. 268-269)

Estos entornes digitales son factibles en Ecuador ya que las universidades como los hogares poseen equipos tecnológicos que permiten la cohesión de las herramientas con los estudiantes y docentes. En la Tabla 1 se puede observar el equipamiento tecnológico y la Tabla 2 el acceso a internet en los hogares ecuatorianos. Aquí se demuestra que hay un incremento de la adquisición de computadora de escritorio o portátil de los ecuatorianos, como también el incremento del acceso a internet en los hogares del Ecuador en los últimos años.

“Hay un 98,28\% de las IES del Ecuador poseen entornos virtuales de enseñanza aprendizaje, el 94,83\% emplea repositorios digitales y el $100 \%$ de las mismas posee acceso a bibliotecas y base de datos científicas, con lo que se demuestra que las universidades han invertido sustancialmente sus recursos para la adquisición y empleo de tecnología para apoyar y solventar el proceso de enseñanza-aprendizaje”. (Moya, 2017, pág. 135) 


\section{Competencias digitales en docentes: desafío de la educación superior}

Vol. 3, núm. 3., (2019)

Carlos Fernando Morales Vera; Lourdes Ximena Reyes Suárez; Mariana Noemi Medina Suarez; Amir Ricardo Villon Cruz

Tabla 1. Equipamiento tecnológico del hogar a nivel nacional

\begin{tabular}{cccccc}
\hline Periodo & $\begin{array}{c}\text { Computadora de } \\
\text { escritorio }\end{array}$ & $\begin{array}{c}\text { Computadora } \\
\text { portátil }\end{array}$ & $\begin{array}{c}\text { Computadora de } \\
\text { escritorio y } \\
\text { portátil }\end{array}$ & Telefonía fija & $\begin{array}{c}\text { Telefonía } \\
\text { celular }\end{array}$ \\
\hline dic-07 & $20,3 \%$ & - & - & $35,5 \%$ & - \\
dic-08 & $22,8 \%$ & - & - & $37,1 \%$ & - \\
dic-09 & $23,4 \%$ & - & - & $35,6 \%$ & - \\
dic-10 & $24,0 \%$ & $9,0 \%$ & $6,0 \%$ & $38,5 \%$ & $49,7 \%$ \\
dic-11 & $24,7 \%$ & $9,8 \%$ & $5,8 \%$ & $39,9 \%$ & $78,8 \%$ \\
dic-12 & $26,4 \%$ & $13,9 \%$ & $8,1 \%$ & $42,4 \%$ & $81,7 \%$ \\
dic-13 & $27,5 \%$ & $18,1 \%$ & $9,9 \%$ & $39,6 \%$ & $86,4 \%$ \\
dic-14 & $27,1 \%$ & $20,6 \%$ & $10,2 \%$ & $38,2 \%$ & $89,7 \%$ \\
dic-15 & $27,7 \%$ & $24,8 \%$ & $11,6 \%$ & $38,9 \%$ & $89,5 \%$ \\
dic-16 & $26,7 \%$ & $27,6 \%$ & $12,0 \%$ & $38,4 \%$ & $90,1 \%$ \\
dic-17 & $25,9 \%$ & $26,0 \%$ & $11,2 \%$ & $36,9 \%$ & $90,7 \%$ \\
\hline
\end{tabular}

Fuente: (ENEMDU, 2018)

Nota: (-) Información no disponible. Computadora portátil incluye laptop y Tablet.

Tabla 2. Porcentaje de hogares que tienen acceso a internet

\begin{tabular}{cccc}
\hline Periodo & Nacional & Urbana & Rural \\
\hline dic-08 & $7,0 \%$ & $9,9 \%$ & $0,9 \%$ \\
dic-09 & $7,7 \%$ & $11,0 \%$ & $0,7 \%$ \\
dic-10 & $11,8 \%$ & $16,7 \%$ & $1,3 \%$ \\
dic-11 & $16,9 \%$ & $23,7 \%$ & $3,5 \%$ \\
dic-12 & $22,5 \%$ & $31,4 \%$ & $4,8 \%$ \\
dic-13 & $28,3 \%$ & $37,0 \%$ & $9,1 \%$ \\
dic-14 & $32,4 \%$ & $40,4 \%$ & $14,6 \%$ \\
dic-15 & $32,8 \%$ & $41,0 \%$ & $13,7 \%$ \\
dic-16 & $36,0 \%$ & $44,6 \%$ & $16,4 \%$ \\
dic-17 & $37,2 \%$ & $46,1 \%$ & $16,6 \%$ \\
\hline
\end{tabular}

Fuente: (ENEMDU, 2018)

Nota: (-) Información no disponible. Computadora portátil incluye laptop y Tablet. 


\section{Competencias digitales en docentes: desafío de la educación superior}

Vol. 3, núm. 3., (2019)

Carlos Fernando Morales Vera; Lourdes Ximena Reyes Suárez; Mariana Noemi Medina Suarez;

Amir Ricardo Villon Cruz

El auge de las redes sociales dentro de la población ha permitido un acercamiento de las personas ubicadas en distintos puntos geográficos. Esta característica es tomada para acercar el conocimiento y empoderar a los individuos del conocimiento. Las redes sociales en las universidades ecuatorianas se constituyen en herramientas de generación y retroalimentación de información, donde resulta eficiente e innovador, aunque todavía es necesario continuar masificando su uso y buscar sus utilidades para incorporarlos a los procesos de aprendizaje(Téllez \& Trujillo, 2018).En la Tabla 3 se visualiza la población ecuatoriana con telefonía celular inteligente y uso de las redes sociales en los últimos años. Esto demuestra el auge de estos medios y por la cual es factible poder desarrollar nuevas técnicas pedagógicas para el desarrollo del conocimiento.

Tabla 3. Población con celular inteligente y redes sociales a nivel nacional

\begin{tabular}{ccccc}
\hline Período & $\begin{array}{c}\text { Población } \\
\text { Total }\end{array}$ & $\begin{array}{c}\text { Población que } \\
\text { tiene teléfono } \\
\text { celular activado }\end{array}$ & $\begin{array}{c}\text { Población que } \\
\text { tiene teléfono } \\
\text { celular } \\
\text { SMARTPHONE }\end{array}$ & $\begin{array}{c}\text { Población que en } \\
\text { su teléfono } \\
\text { smartphone } \\
\text { utiliza redes } \\
\text { sociales }\end{array}$ \\
\hline dic-11 & 14.478 .129 & 6.209 .858 & 522.640 & 365.427 \\
dic-12 & 14.682 .556 & 6.859 .938 & 839.705 & 641.914 \\
dic-13 & 15.872 .755 & 7.453 .781 & 1.261 .944 & 1.081 .620 \\
dic-14 & 16.148 .648 & 7.820 .597 & 1.928 .108 & 1.722 .159 \\
dic-15 & 16.404 .531 & 8.174 .520 & 3.084 .886 & 2.807 .282 \\
dic-16 & 16.714 .929 & 8.482 .236 & 4.484 .087 & 4.224 .984 \\
dic-17 & 16.961 .926 & 8.987 .750 & 5.716 .770 & 5.412 .575 \\
\hline
\end{tabular}

Fuente: (ENEMDU, 2018)

Todo esto genera buen augurio para la masificación del conocimiento y sobre todo para el desarrollo de profesionales que serán la bujía para la economía del país. Es importante la 


\section{Competencias digitales en docentes: desafío de la educación superior}

Vol. 3, núm. 3., (2019)

Carlos Fernando Morales Vera; Lourdes Ximena Reyes Suárez; Mariana Noemi Medina Suarez; Amir Ricardo Villon Cruz

contribución de la innovación al desarrollo de una región el cual depende en gran medida de estimularla en todos los niveles de educación, en especial la universitaria.

“A ello no escapa el Ecuador; país que pretende alcanzar altos índices de calidad en este subsistema educativo, reforzando la institucionalización de los esfuerzos innovadores. Todo esto desde la acción y la reflexión; la aglutinación de los saberes y el fortalecimiento de la profesión docente y en lo cual juega un rol trascendental, como elemento clave en el quehacer práctico-teórico del docente universitario, el portafolio docente".(Salinas, Alcívar, Gómez Cabrera, \& Aguilar Salazar, 2016, pág. 302)

Es por ello, que el docente juega un papel importante para el desarrollo de estas herramientas pedagógicaspor lo que se considera una variable subjetiva ya que depende en gran medida el grado de motivación para poder quebrar los viejos paradigmas y montarse en la ola de la globalización educativa. Por lo que, se considera un desafío para los docentes universitarios las utilizaciones pedagógicas de las tecnologías emergentes y la evaluación de su impacto en el desarrollo integral del estudiante(Téllez \& Trujillo, 2018).

La relación de las TIC y los docentes de la educación superior

Este mundo globalizado ha llevado las tecnologías a las puertas de las instituciones de educación superior por lo cual existe la necesidad formar en competencias digitales a toda la comunidad universitaria. Esto es debido a que las universidades debenofrecer a la ciudadanía una educación superior, donde,entre otras metas, se les forme como sujetos competentespara afrontar los complejos desafíos de la cultura, conocimiento,ciencia, economía y de las relaciones sociales de este siglo XXI(Area Moreira, 2010). 


\section{Competencias digitales en docentes: desafío de la educación superior}

Vol. 3, núm. 3., (2019)

Carlos Fernando Morales Vera; Lourdes Ximena Reyes Suárez; Mariana Noemi Medina Suarez;

Amir Ricardo Villon Cruz

Estos sujetos es la columna vertebral del propósito de las universidades, por lo cual se debe transformar en un nuevo paradigma. La novedad es la contribución a una formación profesional del futuro graduado construida tanto en el ámbito de la universidad como en el de la comunidad, con resultados difíciles de dominar mediante otras estrategias o programas formativos(MARTÍNEZ, 2006).

Para poder lograr esto hay que hacer una fusión entre los viejos métodos pedagógicos con las herramientas digitales. Sólo este tipo de reelaboración, parcialmente consciente, de la base de conocimiento — operando en el contexto de un alcance cada vez más amplio - podría ofrecer más oportunidades para lograr pequeños avances en la concentración del conocimiento(Clark, 1998). Con esto, se podría afirmar que empiezan a existir dos grandes tendencias de las instituciones que imparten la formación de forma virtual, una como actividad complementaria a la presencialidad y otra como actividad formativa con identidad propia(Sangrà, 2003).

No obstante, todos estos esfuerzos no tendrán recompensa si no se cuenta con el compromiso y la complicidad del personal universitario para que finalmente, éste lleve a cabo la integración de las TIC en todas las áreas y ámbitos de la educación superior(BAELO ÁLVAREZ \& CANTÓN MAYO, 2009).Indistintamente, con todos estos planes y objetivos, nuevamente recae el peso es a los docentes universitarios como uno de los garantes que estos nuevos planteamientos se lleven a cabo.

Se debe comenzar a entrelazar esta relación de los docentes con las competencias digitales a través de las diversas herramientas que ofrecen las TIC. Se deben crear plataformas 


\section{Competencias digitales en docentes: desafío de la educación superior}

Vol. 3, núm. 3., (2019)

Carlos Fernando Morales Vera; Lourdes Ximena Reyes Suárez; Mariana Noemi Medina Suarez; Amir Ricardo Villon Cruz

digitales amigables para que los profesores entren en contacto con estas nuevas modalidades evaluativas y de comunicación. Con ello comienza el quiebre de los viejos paradigmas.

"Se espera que, en este nuevo paradigma de formación centrada en el aprendizaje del alumno, los profesores aprovechen en la enseñanza la potencialidad de las TIC, los múltiples recursos disponibles en el ciberespacio y además lo hagan trabajando en colaboración en el seno de comunidades de docentes que participan en la web en tareas de innovación e investigación sobre su propia docencia”.(Díaz Barriga, 2009, pág. 140)

La tecnología en los institutos de educación superior ha ayudado a fomentar nuevos paradigmas. Las universidades públicas ecuatorianas han empezado a dar los primeros pasos los cuales han encontrado trabas que han impedido una mayor velocidad de crecimiento. Entre ellos la infraestructura tecnológica la que no se ha logrado desarrollar de forma óptima, por otro lado, los facilitadores, en este caso los docentes no están preparados para un desarrollo educativo interactivo mediado por las tecnologías(Téllez \& Trujillo, 2018).

Utilizar los recursos en las plataformas web aumenta el espectro de las dimensiones pedagógicas del docente sobre el conocimiento en un área o tema en específico. Es por ello, que la utilización de estas plataformas es primordial para la evolución y desarrollo globalizado de las universidades. La integración de varias herramientas TIC (correo electrónico, Facebook y Twitter) como un sistema de difusión constituye la evolución en los modelos de comunicación universitarios(Cueva, 2016).

"Más allá de un manejo instrumental de las TIC, el docente requiere mejorar y enriquecer las oportunidades de aprender a enseñar significativamente a sus estudiantes con 


\section{Competencias digitales en docentes: desafío de la educación superior}

Vol. 3, núm. 3., (2019)

Carlos Fernando Morales Vera; Lourdes Ximena Reyes Suárez; Mariana Noemi Medina Suarez;

Amir Ricardo Villon Cruz

apoyo en dichas tecnologías. Esto será posible solo en la medida en que el profesorado arribe a formas de enseñanza innovadoras y se forme para participar de manera creativa y autogestiva en el seno de una comunidad educativa que desarrolla una cultura tecnológica”.(Díaz Barriga, 2009, pág. 145)

De estos planos, se conlleva a una realidad que en la mayoría contrasta a estas referencias. En la actualidad la mayoría de docentes utilizan el material didáctico combinándolo de manera empírica con ciertas herramientas tecnológicas que no permiten una mayor enseñanza por lo que requieren capacitaciones continuas en el uso de software y en el uso de las TIC(López \& Constante, 2018). Esto conlleva que los docentes no son el problema sino más bien la solución. No hay buenos ni malos profesores en la integración de las tecnologías de la información en el trabajo educativo, sino que hay profesores con mejor o peor preparación y con adecuado o ausente apoyo y soporte técnico y pedagógico(Martínez Alvarado, 2009).

Por lo tanto, el docente, más allá del rol de gestor y guía, constituye en sí un modelo de educando, que promueve continuamente su propia formación y que participa activamente en escuelas que funcionan como comunidades $u$ organizaciones que aprenden $y$ se transforman(Díaz Barriga, 2009). En la Tabla 4 se puede observar los estándares de competencias en TIC para docentes según la UNESCO. 


\section{Competencias digitales en docentes: desafío de la educación superior}

Vol. 3, núm. 3., (2019)

Carlos Fernando Morales Vera; Lourdes Ximena Reyes Suárez; Mariana Noemi Medina Suarez; Amir Ricardo Villon Cruz

Tabla 4. Estándares de competencias en TIC para docentes según la UNESCO

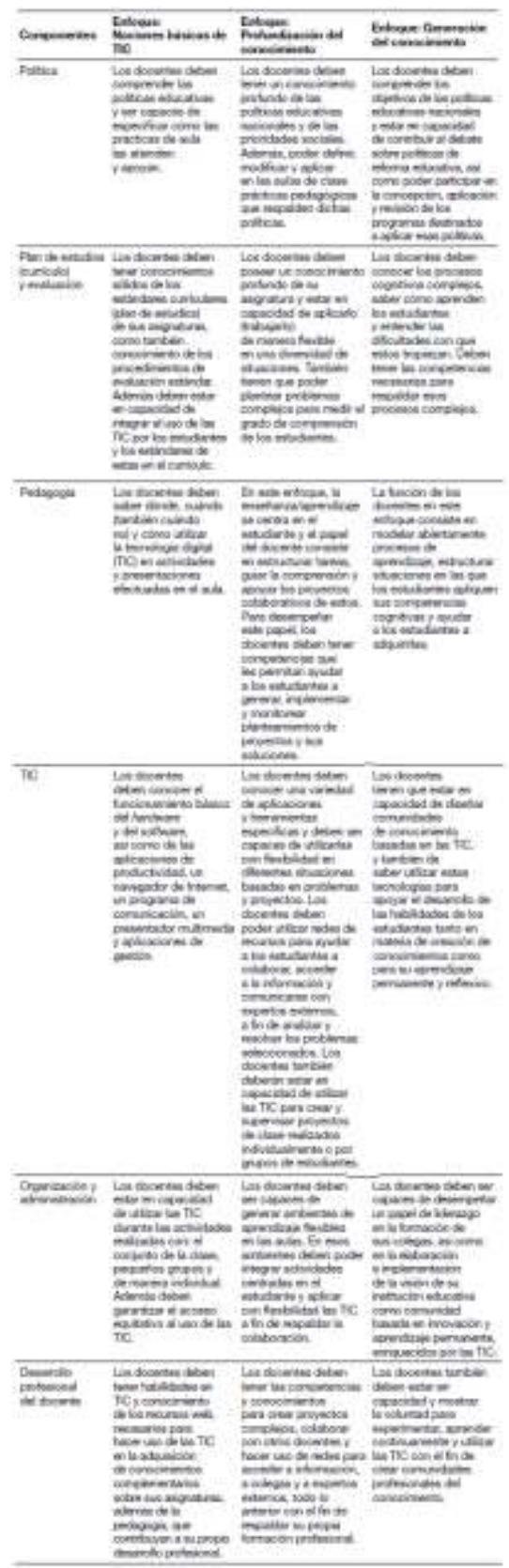

Fuente: (Díaz Barriga, 2009) 


\section{Competencias digitales en docentes: desafío de la educación superior}

Vol. 3, núm. 3., (2019)

Carlos Fernando Morales Vera; Lourdes Ximena Reyes Suárez; Mariana Noemi Medina Suarez;

Amir Ricardo Villon Cruz

\section{Discusión y conclusiones.}

Dentro de los sistemas de educación se han creado formas pedagógicas clásicas que han funcionado debido a que la información ha llegado a los estudiantes permitiendo, analizar y comprender los conceptos e ideas que los conlleve a poder obtener el título profesional. Los docentes forman sus estadísticas y de estas herramientas las transforman en una cultura educativa predominante. Estas formas de construir el conocimiento han dado pie a la formación de paradigmas dentro de la educación. Los mismos incrustados no solo en los docentes sino en todos aquellos que hacen vida en estas instituciones educativas. La educación superior no escapa a tales efectos de estos viejos paradigmas. El miedo al cambio, el conformismo, salir de la zona de confort son los parámetros que rigen estos fenómenos educativos y que conllevan a que la educación y por ende la generación del conocimiento se quede dentro de las aulas de clases como único protagonista el profesor basado en su experiencia docente en función del tiempo de trabajo.

El mundo globalizado que se vive en pleno siglo XXI ha permitido que las tecnologías productivas, de comunicación y de conocimiento avance a pasos gigantes. Es allí donde esta nueva cultura choca de frente ante el muro de los viejos paradigmas. Este choque a sido una compresión constante, y en aumento, frente a la pared que se resiste al cambio inminente que está viviendo la sociedad. Se está formando un nuevo paradigma basado en las tecnologías de la información y comunicación. Todo lo que rodea a la sociedad ya está implicando conocer cómo se maneja y se usa distintas tecnologías que acercan a un más al saber. Las universidades no pueden escapar ante este hecho preciso. Se han empezado a dar los primeros pasos basándose en 


\section{Competencias digitales en docentes: desafío de la educación superior}

Vol. 3, núm. 3., (2019)

Carlos Fernando Morales Vera; Lourdes Ximena Reyes Suárez; Mariana Noemi Medina Suarez; Amir Ricardo Villon Cruz

variables como infraestructura, dotación de equipos y capacitación a los usuarios de estos servicios.

Se han creado diferentes plataformas educativas las cuales se han diseñado para ser amigables, tanto para el docente como para el estudiante, lo cual permite la interacción fuera del aula de clase y con la ayuda de herramientas que permitan una mayor comprensión del conocimiento. Estas herramientas son las redes sociales entre las que se destacan el Facebook, twitter, youtube, instegram, entre otros. Estas permiten un acercamiento más amigable y porque entre ellas se pueden vincular y relacionar trayendo a colación fotos, videos, artículos científicos y cualquier medio que permita poder conocer aún más los conocimientos necesarios. En el Ecuador estas herramientas pueden ser utilizadas ya que la mayoría de la población las domina y tiene acceso a ellas en cualquier momento y espacio. Estas circunstancias las transforman como las nuevas herramientas pedagógicas de vanguardia en la actualidad.

Por lo tanto, la relación que tienen los docentes con estas competencias digitales es muy factible, siempre y cuando se tenga la convicción al cambio por parte del facilitador y que también cuente con el apoyo en equipo e infraestructura por parte de los institutos de educación superior. Aunque ha sido un proceso lento, se ha comenzado con esta transformación de la educación a nivel superior, la cual permitirá la generación de conocimiento de manera rápida y eficiente donde el docente tendrá en su poder apegarse al cambio que se está viviendo en este mundo globalizado. 


\section{Competencias digitales en docentes: desafío de la educación superior}

Vol. 3, núm. 3., (2019)

Carlos Fernando Morales Vera; Lourdes Ximena Reyes Suárez; Mariana Noemi Medina Suarez;

Amir Ricardo Villon Cruz

\section{Bibliografía.}

Acosta, O., \& Genyelbert, E. (2013). Globalización e internacionalización de la educación superior: un enfoque epistemológico. Omnia, 19(1), 75-85.

Area Moreira, M. (2010). ¿Por qué formar en competencias informacionales y digitales en la educación superior? RUSC. Universities and Knowledge Society Journal, vol. 7, núm. 2, $2-5$.

BAELO ÁLVAREZ, R., \& CANTÓN MAYO, I. (2009). Las tecnologías de la información y la comunicación en la educación superior. Estudio descriptivo y de revisión. Revista Iberoamericana de Educación. n. ${ }^{\circ}$ 50/7, 1-12.

Briceño, J. (2014). Reflexiones sobre la educación superior en Venezuela. Comunidad y Salud, 12(1), 69-73.

Cabrera, V. A. (2005). El concepto calidad en la educación universitaria: clave para el logro de la competitividad institucional. Revista iberoamericana de Educación, 36(12), 1-7.

Carrizo Estévez, J. D. (2010). Importancia de la investigación en la formación de Pregrado. Panorama Cuba y Salud, vol. 5, núm. 3, 3-4.

Castro, S. G. (2007). Las Tic en los procesos de enseñanza y aprendizaje. Laurus, vol. 13, núm. 23., 213-234.

Clark, B. R. (1998). Crecimiento sustantivo y organización innovadora: nuevas categorías para la investigación en educación superior. Perfiles Educativos, núm. 81, 1-16.

Coll, C. (2008). Aprender y enseñar con las TIC: expectativas, realidad y potencialidades. Boletín de la Institución Libre de Enseñanza, 72, 17-40.

Cueva, M. C. (2016). Las Tecnologías de la Información y la Comunicación como Medio de Difusión Universitaria. Hallazgos21, develando ciencia por el desarrollo, 1(2), 172-180.

Díaz Barriga, F. (2009). TIC y competencias docentes del siglo XXI. En R. Carneiro, J. C. Toscano, \& T. Díaz, Los desafíos de las TIC para el cambio educativo. (págs. 139-154). Madrid, España: Fundación Santillana.

ENEMDU. (2018). Tecnologías de la Información y Comunicación. Quito - Ecuador: Instituto Nacional de Estadística y Censos. 


\section{Competencias digitales en docentes: desafío de la educación superior}

Vol. 3, núm. 3., (2019)

Carlos Fernando Morales Vera; Lourdes Ximena Reyes Suárez; Mariana Noemi Medina Suarez; Amir Ricardo Villon Cruz

Escofet Roig, A., García Gonzalez, I., \& Gros Salvat, B. (2011). Las nuevas culturas de aprendizaje y su incidencia en la educación superior. Revista mexicana de investigación educativa, 16(51), 1177-1195.

Facundo, Á. H. (2004). La virtualización desde la perspectiva de la modernización de la educación superior: consideraciones pedagógicas. International Journal of Educational Technology in Higher Education (ETHE), 1(1), 1-17.

GIL PEREZ, D. (1983). TRES PARADJGMAS BASICOS EN LA ENSENANZA DE LAS CIENCIAS. ENSENANZA DE LAS CIENCIAS, 26-33.

Graells, P. M. (2013). Impacto de las TIC en la educación: funciones y limitaciones. 3C TIC, 2(1), 1-15.

Gros, B., \& Noguera, I. (2015). Mirando el futuro: evolución de las tendencias tecnopedagógicas en educación superior. Campus virtuales, 2(2), 130-140.

Guanoluisa, D. M., \& Herrera, L. A. (2018). Estudio de innovación tecnológica aplicada en procesos formativos con sistemas de gestión de aprendizaje (LMS) en la educación universitaria del Ecuador. Revista Ibérica de Sistemas e Tecnologias de Informação, (E15), 256-270.

López Noguero, F. (2002). El análisis de contenido como método de investigación. Revista de Educación - Universidad de Huelva, 13., 167-179.

López, Y. A., \& Constante, G. F. (2018). Las TIC como herramienta de apoyo didáctico en el proceso de enseñanza-aprendizaje. Revista Científica Cátedra, 1(1), 29-31.

Martínez Alvarado, H. (2009). La integración de las TIC en instituciones educativas. En R. Carneiro, Los desafíos de las TIC para el cambio educativo (págs. 61-70). Madrid, España: Fundación Santillana.

MARTÍNEZ, M. (2006). FORMACIÓN PARA LA CIUDADANÍA Y EDUCACIÓN SUPERIOR. REVISTA IBEROAMERICANA DE EDUCACIÓN. N. ${ }^{\circ}$ 42, 85-102.

Milia, M. F. (2014). Marco de Políticas Públicas de Ciencia, Tecnología y Educación Superior en el Ecuador. Nuevos horizontes: dinámicas y condicionamientos para una Investigación Universitaria de cara a la Sociedad. Buenos Aires, Argentina.: Congreso Iberoamericano de Ciencia, Tecnología, Innovación .

Mora, J. G. (2004). La necesidad del cambio educativo para la sociedad del conocimiento. Revista Iberoamericana de educación, 35(2), 13-37. 


\section{Competencias digitales en docentes: desafío de la educación superior}

Vol. 3, núm. 3., (2019)

Carlos Fernando Morales Vera; Lourdes Ximena Reyes Suárez; Mariana Noemi Medina Suarez;

Amir Ricardo Villon Cruz

Moya, E. J. (2017). Utopía o realidad de aplicaciones informáticas en la educación. Caso Universidad Ecuatoriana. Revista Publicando, 3(9), 119-137.

Ortí, C. B. (2011). Las tecnologías de la información y comunicación (TIC). Univ. Val., Unidad Tecnol. Educ, (951), 1-7.

Ortiz Flores, E. P., \& Bernal Zepeda, M. (2007). Importancia de la incorporación temprana a la investigación científica en La Universidad de Guadalajara. Guadalajara, México.

Pereira Pérez, Z. (2011). Los diseños de método mixto en la investigación en educación: Una experiencia concreta. Revista Electrónica Educare, vol. XV, núm. 1, 15-29.

Post, D. (2011). Las reformas constitucionales en el Ecuador y las oportunidades para el acceso la educación superior desde 1950. Education Policy Analysis Archives/Archivos Analíticos de Políticas Educativas, 19, 1-24.

Quintero, L. J. (2009). Las universidades apostando por las TIC: modelos y paradojas de cambio institucional. EDUTEC. Revista Electrónica de Tecnología Educativa, (28), 1-14.

Rama, C. (2010). La irrupción de nuevos modelos socioeconómicos, paradigmas educativos y lógicas económicas de la educación. Universidades, 60(46), 3-16.

Ramírez, R., \& Minteguiada, A. (2010). Transformaciones en la Educación Superior Ecuatoriana: Antecedentes y perspectivas futuras como consecuencias de la nueva constitución política. Revista Educación Superior y Sociedad (ESS) ISSN: 0798-1228, 15(1), 129-154.

Ramos, C. A. (2015). Los paradigmas de la investigación científica. Av.psicol. 23(1), 9-17.

Reig-Hernández, D. (2010). El futuro de la educación superior, algunas claves. REIRE. Revista d'Innovació i Recerca en Educació, 3(2), 98-113.

Rodríguez-Izquierdo, R. M. (2011). Repensar la relación entre las TIC y la enseñanza universitaria: problemas y soluciones. Profesorado. Revista de curriculum y formación del profesorado, 9-22.

Salinas, Q., Alcívar, L., Gómez Cabrera, O. A., \& Aguilar Salazar, R. D. (2016). La innovación educativa en la Educación Superior ecuatoriana y el portafolio docente: instrumentos de desarrollo. Revista Cubana de Reumatología, 18(3), 297-303.

Sangrà, A. (2003). La calidad en las experiencias virtuales de educación superior. Barcelona, España: Universitat Oberta de Catalunya (UOC). 


\section{Competencias digitales en docentes: desafío de la educación superior}

Vol. 3, núm. 3., (2019)

Carlos Fernando Morales Vera; Lourdes Ximena Reyes Suárez; Mariana Noemi Medina Suarez; Amir Ricardo Villon Cruz

Sunkel, G. (2009). Las TIC en la educación en América Latina: visión panorámica. En R. Carneiro, J. C. Toscano, \& T. Díaz, Los desafíos de las TIC para el cambio educativo. (págs. 29-43). Madrid, España: Fundación Santillana.

Tamayo, C. F. (2012). Plataformas virtuales como recurso para la enseñanza en la universidad: análisis, evaluación y propuesta de integración de moodle con herramientas de la web 2.0. Madrid, Españas: Trabajo de Grado - Universidad Complutense de Madrid.

Téllez, M. N., \& Trujillo, E. P. (2018). Las redes sociales en el contexto académico universitario. Desafios al docente.Etic@net, 18(1),83-101.

Zaccagnini, M. C. (2003). Impacto de los paradigmas pedagógicos históricos en las prácticas educativas contemporáneas. Revista Iberoamericana de Educación, 33(2), 1-29.

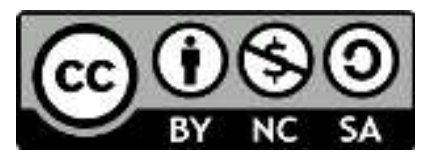

RECONOCIMIENTO-NOCOMERCIAL-COMPARTIRIGUAL

CC BY-NC-SA

ESTA LICENCIA PERMITE A OTROS ENTREMEZCLAR, AJUSTAR Y CONSTRUIR A PARTIR DE SU OBRA CON FINES NO

COMERCIALES, SIEMPRE Y CUANDO LE RECONOZCAN LA AUTORÍA Y SUS NUEVAS CREACIONES ESTÉN BAJO UNA LICENCIA CON LOS MISMOS TÉRMINOS. 